\title{
Expression of Sweet Potato Senescence- Associated Cysteine Proteases Affect Seed and Silique Development and Stress Tolerance in Transgenic Arabidopsis
}

\author{
Hsien-Jung Chen ${ }^{1}$, Guan-Jhong Huang ${ }^{2}$, Chia-Hung Lin ${ }^{1}$, \\ Yi-Jing Tsai ${ }^{1}$, Zhe-Wei Lin ${ }^{1}$, Shu-Hao Liang ${ }^{1}$ and Yaw-Huei Lin ${ }^{3}$ \\ ${ }^{1}$ Department of Biological Sciences, National Sun Yat-sen University, Kaohsiung \\ ${ }^{2}$ School of Chinese Pharmaceutical Sciences and Chinese Medicine Resources, \\ College of Pharmacy, China Medical University, Taichung \\ Institute of Plant and Microbial Biology, Academia Sinica, Nankang, Taipei
}

Taiwan

\section{Introduction}

Leaf is in general the main site of photosynthesis and acts as a carbohydrate source for nutrients to support the growth in sink organs of plants. Therefore, its longevity and senescent level may affect the photosynthesis efficiency and thus crop yield. There are endogenous and exogenous factors affecting leaf senescence, including plant growth regulators, sucrose starvation, dark, cold, drought, salt, wound, pathogen infection and insect attack (Yoshida, 2003; Lim et al., 2007). Leaf senescence is the final developmental stage of leaves and has been considered as a type of programmed cell death. During leaf senescence, it is not only a degradative process but also a recycling one. Therefore, macromolecules and organelles can be degraded into small molecules, salvaged and mobilized from the senescent cells to other sinks, such as young leaves, developing seeds, or storage tissues (Buchanan-Wollaston, 1997; Quirino et al., 2000).

In sweet potato, several morphological, biochemical and physiological changes have also be observed during leaf senescence, including leaf yellowing, decrease of chlorophyll contents, reduction of photochemical $\mathrm{Fv} / \mathrm{Fm}$, elevation of $\mathrm{H}_{2} \mathrm{O}_{2}$ amount, increase of plastoglobuli number in chloroplast, activation of senescence-associated gene expression, and finally cell death (Chen et al., 2000; Chen et al., 2003; Chen et al. 2010a). Several full-length cDNAs encoding putative isocitrate lyase, papain-like cysteine proteases and asparaginyl endopeptidase, have been cloned from senescent leaves (Chen et al., 2000, 2004, 2006, 2008, 2009, 2010b), which likely play roles in association with lipid degradation and gluconeogenesis, and protein degradation and re-mobilization. These data support the occurrence of macromolecule and organelle degradation into small molecules for recycling and re-mobilization during sweet potato leaf senescence.

During senescence, breakdown of leaf proteins by proteases provides a large pool of cellular nitrogen for recycling (Makino \& Osmond, 1991). In plants, different degradation pathways 
have been described and the vacuolar degradation pathway is assumed to be involved in bulk protein degradation by virtue of the resident proteases in the vacuole (Vierstra, 1996). There are two types of vacuoles described in plants: the storage vacuole and the lytic vacuole (Marty, 1999). Protein storage vacuoles are found in seed tissues and accumulate proteins that are re-mobilized and used as the main nutrient resource for germination (Senyuk et al., 1998; Schlereth et al., 2001). Most cells in vegetative tissues have lytic vacuoles, containing a wide range of proteases in an acidic environment. Substrate proteins must be transported and sequestered into these lytic vacuoles before degradation. Therefore, senescence-associated vacuoles are lytic vacuoles and involved in the degradation of imported chloroplast proteins in tobacco leaves (Martı́nez et al., 2008).

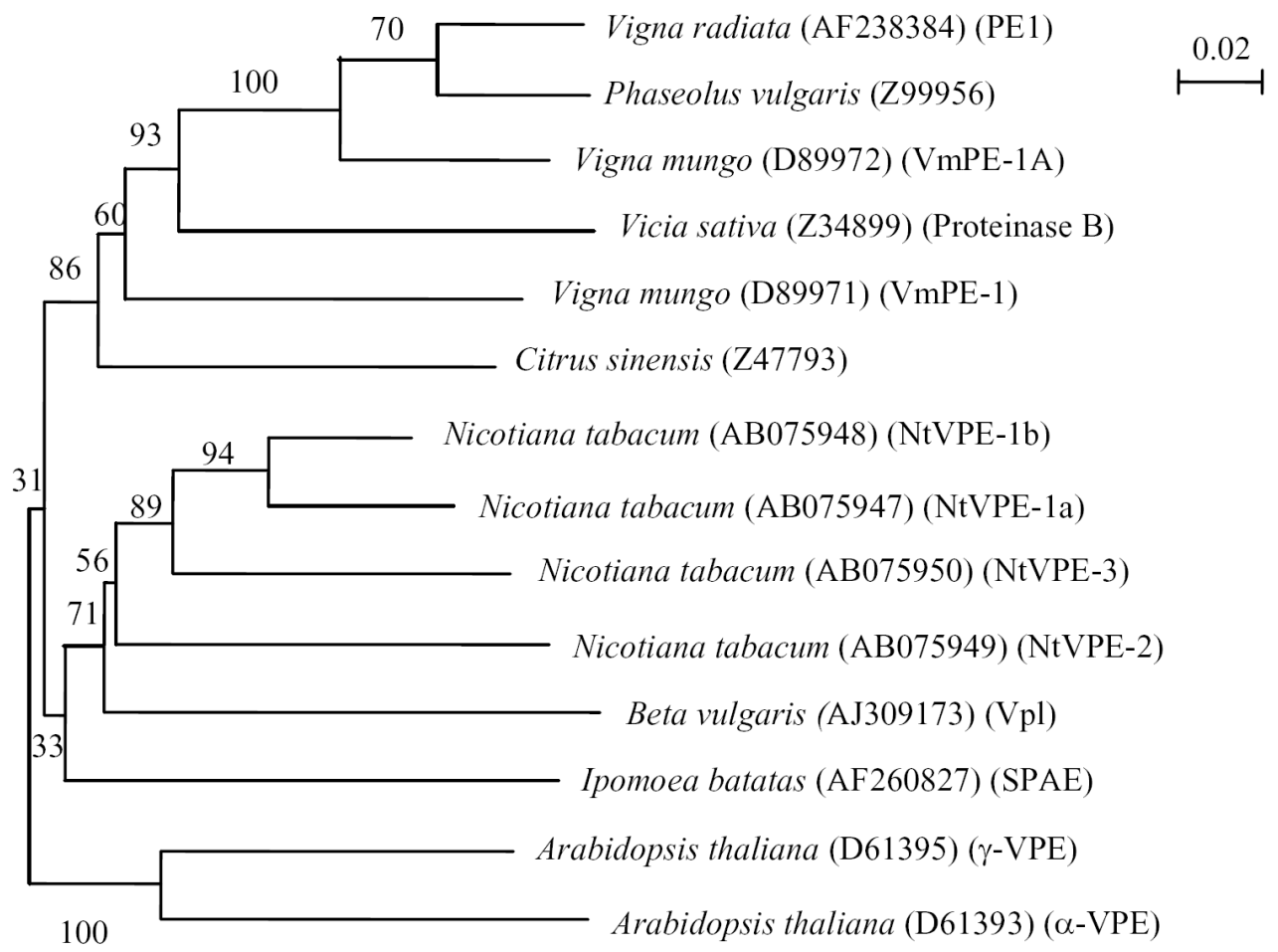

Fig. 1. Phylogenetic tree analysis of plant asparaginyl endopeptidases (Adapted and Modified from Chen et al., 2004).

The molecular mechanisms for vacuolar protein degradation and nutrient recycling pathway in senescent leaves are generally not clear. Phylogenetic tree analysis indicated that sweet potato asparaginyl endopeptidase (SPAE) exhibited high amino acid sequence identities and closely-related association with plant vacuolar processing enzymes (VPEs) or legumains, including legumain-like protease LLP of kidney bean (Phaseolus vulgaris), legumain-like protease VsPB2 of vetch (Vicia sativa), vacuolar processing enzymes of Arabidopsis thaliana, and asparaginyl endopeptidases VmPE-1 of Vigna mungo (Fig. 1). Sweet potato papain-like cysteine protease (SPCP2) also showed high amino acid sequence 
identities and closely-related association with a subgroup of cysteine proteases, including Actinidia deliciosa CP3, Arabidopsis thaliana RD19, Brassica oleracea BoCP4, Phaseolus vulgaris CP2, Solanum melongena SmCP, Vicia sativa CPR2, and Vigna mungo SH-EP (Fig. 2). These data suggest the possible physiological roles and functions for SPAE and SPCP2 related to these mentioned vacuolar processing enzymes and papain-like cysteine proteases, respectively.

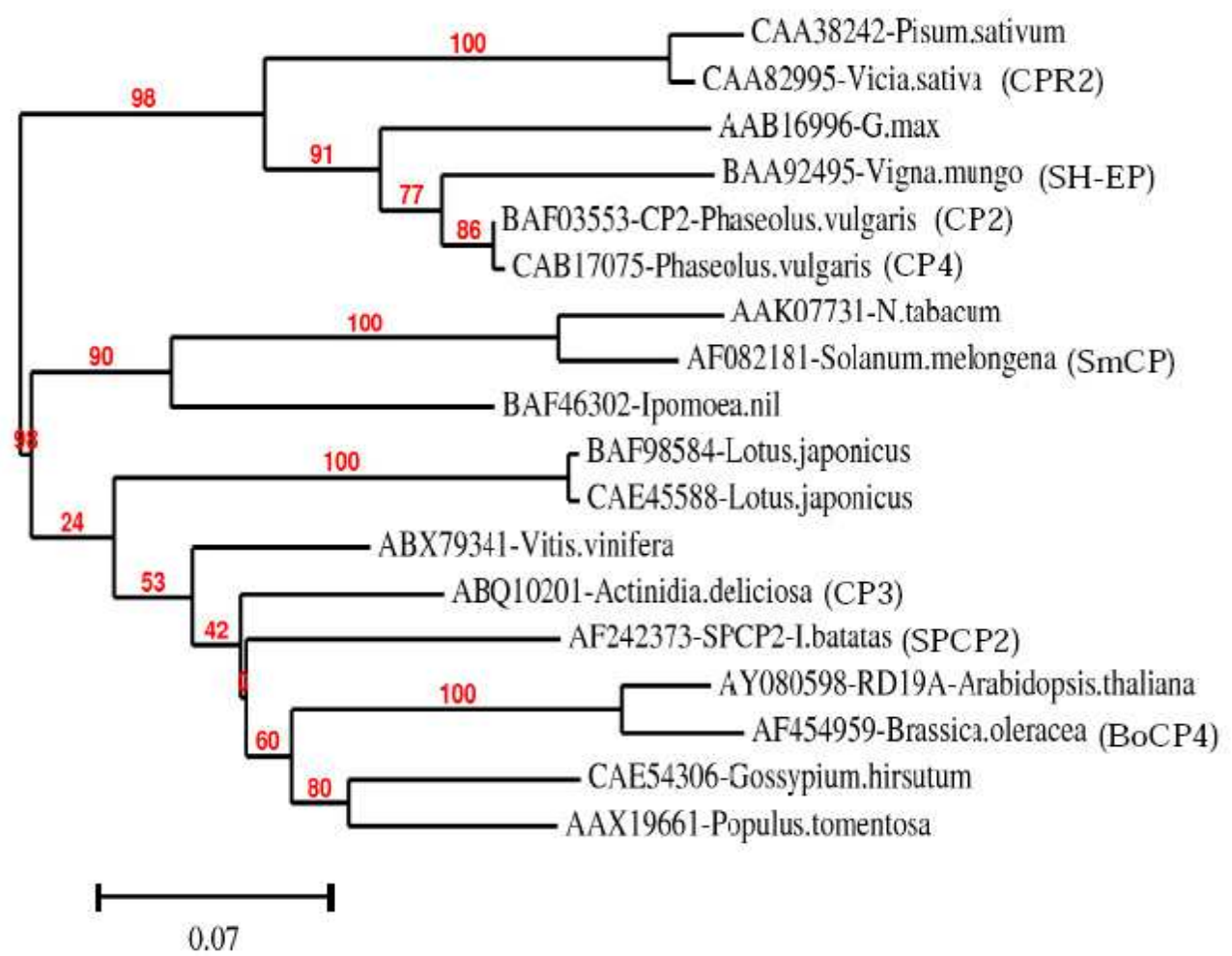

Fig. 2. Phylogenetic tree analysis of plant papain-like cysteine proteases (Adapted from Lin, 2010).

\section{Association of vacuolar processing enzyme and papain-like cysteine protease with seed storage globulin protein degradation}

Vacuolar processing enzyme is a novel group of cysteine endopeptidase and has recently been found in seeds. The enzyme exhibits strict cleavage specificity for the peptide bonds of seed globulin storage proteins with asparagines at the P1 position, and is called asparaginyl endopeptidase (Ishii, 1994). The substrate specificity was observed with purified asparaginyl endopeptidases from developing seeds of castor bean (Hara-Nishimura et al., 1991) and soybean (Scott et al., 1992; Hara-Nishimura et al., 1995), from mature seeds of jack bean (Abe et al., 1993), and from germinating seeds of vetch (Becker et al., 1995). Many seeds accumulate protein reserves in the storage vacuoles, and a number of these proteins 
undergo proteolytic cleavage, including the 7S and 11S seed storage globulins (Müntz \& Shutov, 2002). The 11S seed globulin storage proteins are synthesized as precursors and are cleaved post-translationally in storage vacuoles by an asparaginyl endopeptidase during seed development (Ishii, 1994). In castor bean and soybean seeds, vacuolar processing enzymes were found in the protein bodies and likely associated with the conversion of proproteins into their corresponding mature forms in vacuoles (Hara-Nishimura et al., 1991; Shimada et al., 1994).

Asparaginyl endopeptidases also play a role with bulk degradation and mobilization of storage proteins during seed germination and seedling growth. For example, the asparaginyl endopeptidase, which was also called "legumain-like proteinase" (LLP), was isolated from cotyledons of kidney bean (Phaseolus vulgaris) seedlings. It was the first proteinase ever known which in vitro extensively degraded native phaseolin, the major storage globulin of this grain legume (Senyuk et al., 1998). In vetch (Vicia sativa) seeds, the legumain-like VsPB2 and proteinase B together with additional papain-like cysteine proteinases were responsible for the bulk breakdown and mobilization of storage globulins during seed germination (Schlereth et al., 2000). In Arabidopsis, the seed protein profiles were compared between the wild type and a seed-type vacuolar processing enzyme $\beta V P E$ mutant using a two dimensional gel/mass spectrometric analysis. A significant increase in accumulation of several legumin-type globulin propolypeptides was found in $\beta V P E$ mutant seeds (Gruis et al., 2002).

For papain-like cysteine protease, the vacuolar SH-EP is synthesized in cotyledons of germinated Vigna mungo seeds and is responsible for the degradation of seed globulin storage proteins accumulated in protein bodies. In Vicia faba (vetch), globulins such as legumin and vicillin are major seed storage proteins present in the protein bodies of cotyledon, radicle, axis, and shoots. Papain-like cysteine protease such as CPR2 and CPR4 are found in cotyledon and axis of dry and imbibed seeds. Gene expression studies concluded that storage globulin mobilization in germinating vetch seeds is started by the stored cysteine proteases (CPRs), however, the bulk globulin mobilization is mediated by de novo synthesized CPRs (Schlereth et al., 2000; Schlereth et al., 2001; Tiedemann et al., 2001). These data suggest that papain-like cysteine proteases may play physiological roles and functions in association with seed storage globulin protein degradation and mobilization during seed germination and seedling growth. In addition to the possible physiological function with seed storage globulin protein degradation, papain-like cysteine proteases have also been implied to play a role in cope with environmental cues. For example, a dehydration-responsive papain-like cysteine protease RD19 was cloned and results showed that its expression was strongly induced under high-salt and osmotic stress conditions, which suggests a possible physiological role of RD19 in association with the regulation of plant cell osmotic potential in Arabidopsis thaliana (Koizumi et al., 1993; Xiong et al., 2002). In broccoli, the florets showed water loss during post-harvest storage. Gene expression of papain-like cysteine proteases BoCP4, which exhibited high amino acid sequence identity with Arabidopsis RD19, was also found to be dehydration-responsive and was repressed by water and sucrose (Coupe et al., 2003).

Many vacuolar enzymes are synthesized as pro-proteins and become active after proteolytically processed. In seed storage tissues, specific endoplasmic reticulum (ER)derived compartments containing precursors of cysteine proteases have been described 
(Chrispeels \& Herman, 2000; Toyooka et al., 2000; Hayashi et al., 2001; Schmid et al., 2001). Germination of the seeds induces the expression and processing of those proteases into the mature active forms, which in turn participate in the degradation of cellular materials in storage tissues and provide nutrients to the growing embryo. The mechanism of asparaginyl endopeptidases (VmPE-1) and papain-like cysteine protease (SH-EP) associated with bulk seed storage globulin protein degradation has been studied in Vigna mungo. The vacuolar cysteine protease SH-EP is synthesized in cotyledons of germinated Vigna mungo seeds with an N-terminal and a C-terminal prosegments (Okamoto \& Minamikawa, 1999; Okamoto et al., 1999). Okamoto \& Minamikawa (1995) isolated a processing enzyme, designated VmPE1. VmPE-1 is a member of the asparaginyl endopeptidases and is involved in the posttranslational processing of SH-EP. In addition, the cleavage sites of the in vitro processed intermediates and the mature form of SH-EP were identical to those of SH-EP purified from germinated cotyledons of $V$. mungo. Therefore, it is proposed that the asparaginyl endopeptidase (VmPE-1)-mediated processing functions mainly in the activation of proSHEP during seed germination (Okamoto et al., 1999). The activated SH-EP plays a major role in the degradation of seed storage proteins accumulated in cotyledonary vacuoles of Vigna mungo seedlings (Mitsuhashi et al., 1986). These reports demonstrate a role of asparaginyl endopeptidase associated with papain-like cysteine protease in the bulk breakdown and mobilization of storage globulins during seed germination.

\section{Characterization of sweet potato asparaginyl endopeptidase SPAE and papain-like cysteine protease SPCP2}

Recently, similar compartments have also been described in vegetative tissues of Arabidopsis (Hayashi et al., 2001). These precursor protease vesicles derived from ER are plant specific compartments and contain vesicle-localized vacuolar processing enzyme ( $\gamma \mathrm{VPE})$ precursor, which is critical for maturation of the vacuolar protease AtCPY. The vacuolar protease AtCPY in turn participates in the degradation of cellular components including vacuolar invertase AtFruct4 and various proteins in organs undergoing senescence in Arabidopsis (Rojo et al., 2003). A mechanism of senescence-induced activation of vesicle-localized vacuolar processing enzyme precursor by releasing its inactive form from the precursor protease vesicle into the acidic lumen of the vacuole is suggested. This activation triggers the processing of downstream proteases for protein degradation and recycling in senescing tissues (Rojo et al., 2003). These data suggest sweet potato asparaginyl endopeptidase SPAE and papain-like cysteine protease SPCP2 may also play roles with functions related to protein degradation for nutrient remobilization during leaf senescence.

\subsection{SPAE}

SPAE had been cloned from senescent leaves with PCR-selective subtractive hybridization and exhibited high amino acid sequence homologies to seed vacuolar legumains/asparaginyl endopeptidases of kidney bean (Phaseolus vulgaris), spring vetch (Vicia sativa) and jack bean (Canavalia ensiformis) (Chen et al., 2004). The conserved catalytic residues (His and Cys) and central $\beta$-strands that supported the catalytic residues of human and mouse legumains (Chen et al., 1998) were also found in SPAE, plant legumain/asparaginyl endopeptidase, vacuolar processing enzymes, and the other cysteine proteases (Chen et al., 2004). 
Asparaginyl endopeptidase SPAE encoded a pre-proprotein precursor, which contained a putative mature protein (325 amino acid residues) and an N-glycosylation site at its Cterminus. The deduced molecular mass of mature SPAE protein was, thus, likely between 33 and $36 \mathrm{kDa}$ that detected by protein gel blot with polyclonal antibody against putative SPAE protein (Chen et al., 2004). Asparaginyl endopeptidase is an atypical cysteine endopeptidase with a reported insensitivity to the inhibitor L-3-carboxy-2,3-trans-epoxypropionyl-leucylamino(4-guanidino)butane (E-64) (Okamoto \& Minamikawa, 1999). A cysteine protease activity band with a molecular mass near $36 \mathrm{kDa}$ similar to the protein gel blot results was also detected and exhibited insensitivity to E-64 inhibitor (Chen et al., 2004). These data provide indirect evidence to support the existence of asparaginyl endopeptidase in senescent leaves.

In sweet potato, SPAE gene expression level is higher in dark- or ethephon-treated leaves similar to that in natural senescent leaves. Hormones such as jasmonic acid (JA) and abscisic acid (ABA) also caused the decrease of chlorophyll contents in treated leaves; whereas, did not significantly alter SPAE gene expression level compared to that of untreated dark control in mature green leaves within a 3-day period (Chen et al., 2004). These data suggest that SPAE is a senescence-associated gene and its expression in natural or induced senescent leaves is likely controlled by ethylene, but not by JA and ABA.

\subsection{SPCP2}

SPCP2 had been cloned from senescent leaves with PCR-selective subtractive hybridization. The open reading frame of SPCP2 contained 1101 nucleotides (366 amino acids) and exhibited high amino acid sequence identities with a subgroup of vacuolar cysteine proteases including Actinidia deliciosa CP3, Arabidopsis thaliana RD19, Brassica oleracea BoCP4, Phaseolus vulgaris CP2, Vicia sativa CPR2, and Vigna mungo SH-EP (Chen et al., 2010). These data suggest an intracellular localization of SPCP2 in the vacuole. For SH-EP, a C-terminal KDEL sequence (endoplasmatic retention signal) was proved to be associated with its vacuole-targeting (Okamoto et al., 2003). However, no significant C-terminal KDEL sequence was found for SPCP2. For RD-19, a vacuolar localization was also suggested. However, it can be re-localized to nucleus in the presence of PopP2, an avirulent gene product of $R$. solanacearum (Bernoux et al., 2008; Poueymiro \& Genin, 2009). Therefore, it is possible to assume that different vacuolar targeting mechanisms and signal peptides are involved and associated with different related cysteine protease genes.

SPCP2 gene expression was enhanced in natural senescent leaves and can be induced by dark, ethephon, ABA and JA (Chen et al., 2010). Buchanan-Wollaston et al. (2005) analyzed gene expression patterns and signal transduction pathways of senescence in Arabidopsis induced by different factors. Transcriptome analysis demonstrated that pathways such as dark, ethylene, and JA are all required for gene expression during developmental senescence. Genes associated with essential metabolic processes such as degradation of proteins and peptides and nitrogen mobilization can utilize alternative pathways for induction (Buchanan-Wallaston et al., 2005). Therefore, a possible explanation which is likely associated with multiple signal transduction pathways is suggested for the induction of sweet potato SPCP2 gene expression by different factors, including development, dark, ABA, ethephon and JA. 


\section{Ectopic expression of asparaginyl endopeptidase SPAE and papain-like cysteine protease SPCP2 in transgenic Arabidopsis}

Sweet potato full-length cDNAs of asparaginyl endopeptidase SPAE and papain-like cysteine protease SPCP2 were individually constructed in the T-DNA portion of recombinant $\mathrm{pBI} 121$ vector under the control of $C a M V 35 S$ promoter for transformation of Arabidopsis with Agrobacterium-mediated floral dip transformation method (Clough \& Bent, 1998). Transgenic Arabidopsis plants ectopically expressing sweet potato asparaginyl endopeptidase SPAE (Chen et al., 2008) or papain-like cysteine protease SPCP2 (Chen et al., 2010) were produced, identified and characterized.

\subsection{Expression of sweet potato asparaginyl endopeptidase SPAE altered seed and silique development in transgenic Arabidopsis}

Three transgenic Arabidopsis plants were isolated and identified with floral dip transformation method (Clough \& Bent, 1998). Genomic PCR and protein gel blot analysis confirmed that these Arabidopsis plants (YP1, YP2 and YP3) were transgenic and sweet potato SPAE gene was expressed and properly processed into mature form with a predicted molecular mass near $36 \mathrm{kDa}$ (Chen et al., 2008). Similar results have also been observed and reported for various plant vacuolar processing enzymes, including Vigna mungo VmPE-1 (Okamoto et al., 1999), Arabidopsis $\beta$ VPE (Gruis et al., 2002), Arabidopsis $\gamma$ VPE (Kuroyanagi et al., 2002; Rojo et al., 2003). These data suggest that transgenic Arabidopsis plants may use similar mechanisms for sweet potato SPAE processing, and thus can produce mature sweet potato SPAE protein products.

Transgenic Arabidopsis plants exhibited earlier floral transition from vegetative growth and leaf senescence (Chen et al., 2008). Early transition of vegetative phase to reproductive phase has been considered as a type of senescence. The reasons and mechanisms that sweet potato $S P A E$ gene expression can promote earlier floral transition and enhance senescence in transgenic Arabidopsis plants are not clear. However, Raper et al. (1988) and Rideout et al. (1992) hypothesized that floral transition is stimulated by an imbalance in the relative availability of carbohydrate and nitrogen in the shoot apical meristem. Barth et al. (2006) suggest that the flowering phenotype is likely linked to the endogenous ascorbic acid content. Degradation and removal of flowering repressor(s) by ectopic SPAE expression in transgenic Arabidopsis plants provides another possibility.

Expression of sweet potato SPAE in transgenic Arabidopsis plants caused altered development of seed and silique, elevated percentage of incompletely-developed siliques, and fewer silique numbers per plant than that of control (Figs. 3 and 4). The reasons for altered phenotypic characteristics in transgenic Arabidopsis by sweet potato SPAE expression are not clear. However, sweet potato SPAE is in close association with plant vacuolar processing enzymes of seeds from phylogenetic analysis (Chen et al., 2004). Vacuolar processing enzymes have been reported to be in association with the degradation and mobilization of globulin storage proteins during seed germination and seedling growth in Phaseolus vulgaris (Senyuk et al., 1998), Vigna mungo (Okamoto et al., 1999), Vicia sativa (Schlereth et al., 2000; Schlereth et al., 2001), and Arabidopsis thaliana (Gruis et al., 2002). In Vigna mungo, VmPE-1 has been demonstrated to increase in the cotyledons of germinating seeds and was involved in the post-translational processing of a vacuolar cysteine endopeptidase, designated SH-EP, which degraded seed storage proteins (Okamoto \& 
Minamikawa, 1999). A possible explanation that inappropriate pre-degradation of globulintype storage protein during seed development and maturation by constitutively expressed sweet potato SPAE in transgenic Arabidopsis is suggested. The inappropriate predegradation of globulin-type storage protein may result in partial repression of seed and silique development which in turn leads to higher incompletely-developed silique percentage and lower silique numbers per plant. These data also suggest that sweet potato asparaginyl endopeptidase SPAE may have enzymatic function similar to seed vacuolar processing enzymes for protein degradation and nutrient recycling during leaf senescence.
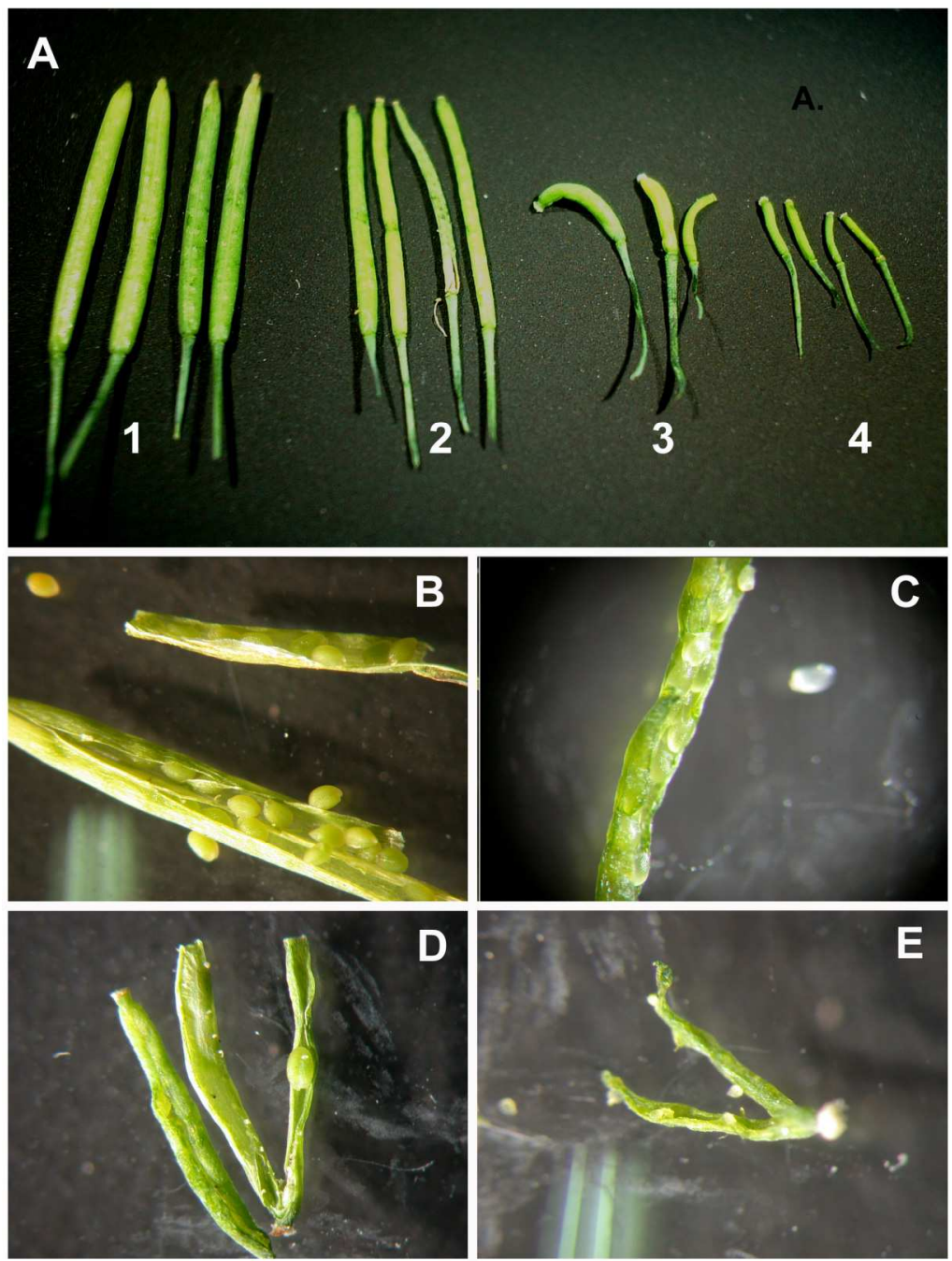

Fig. 3. Morphological classification of Arabidopsis siliques. A. Different silique types (types 1, 2, 3 and 4) classified. B. Dissection of type 1 silique; C. Dissection of type 2 silique; D.

Dissection of type 3 silique; E. Dissection of type 4 silique (Adapted from Chen et al., 2008). 


\section{A. Number of siliques per plant}

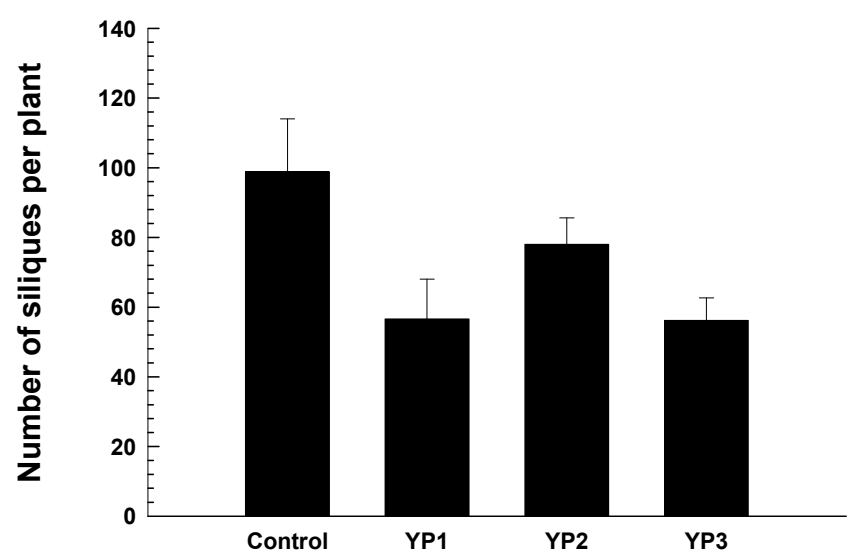

Control and T1 transgenic plants

\section{B. Percentage of incomplete silique development}

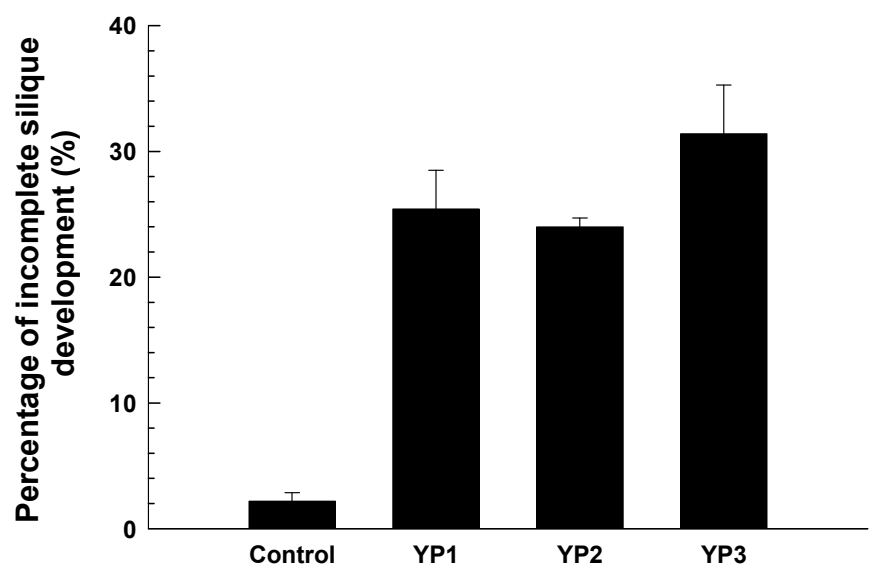

\section{Control and T1 transgenic plants}

Fig. 4. Comparison of silique number per plant and incompletely-developed silique percentage among control and transgenic T1 plants ectopically expressing sweet potato $S P A E$. A. Comparison of silique number per plant. B. Comparison of incompletelydeveloped silique percentages. C and YP1/YP2/YP3 denote non-transformant control and transgenic Arabidopsis plants, respectively. The data are from the average of 5 plants per treatment and shown as mean \pm S.E. (Adapted from Chen et al., 2008). 

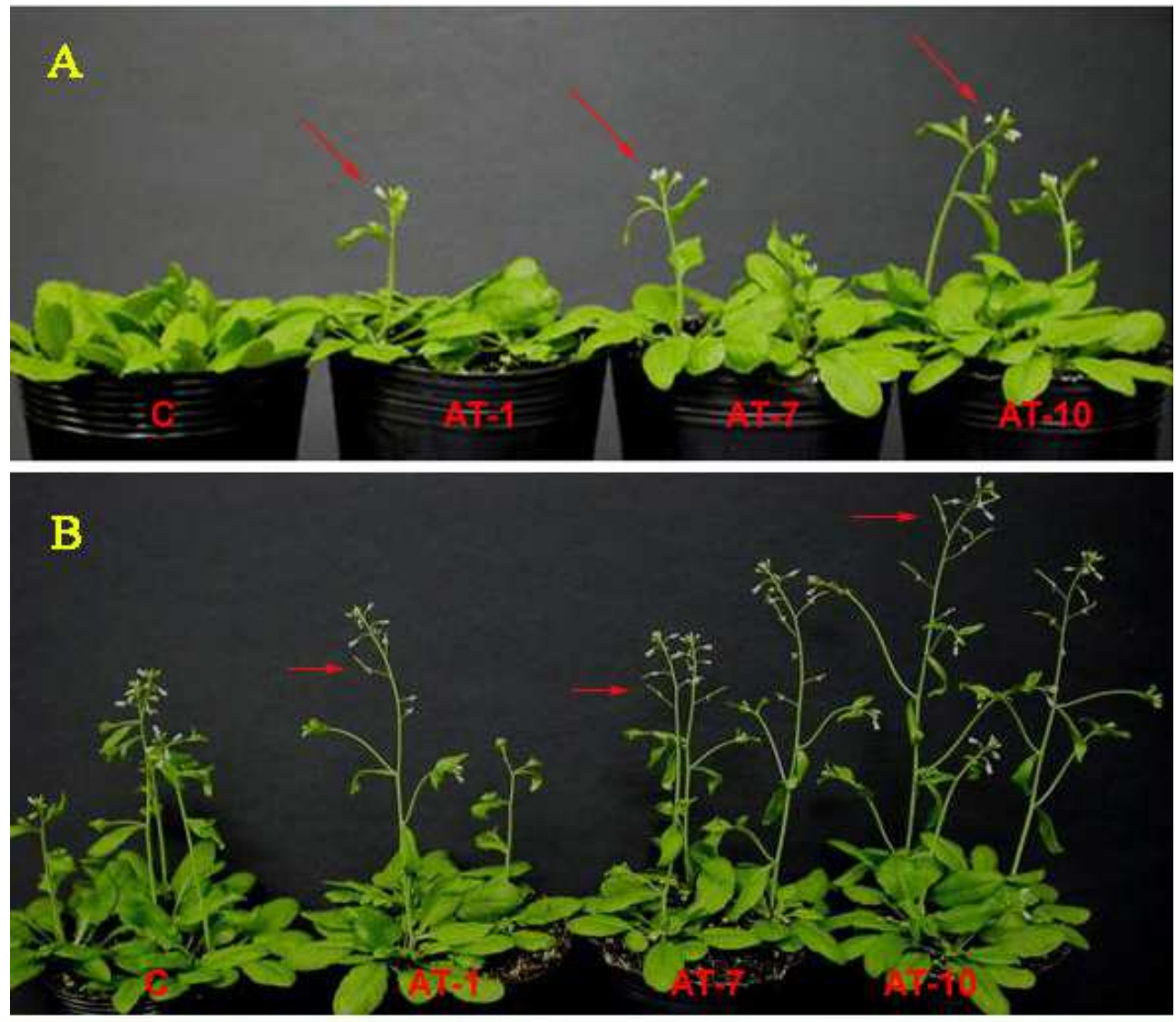

C

$1 \mathrm{~Kb}$

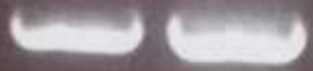

Fig. 5. Comparison of growth patterns among control and transgenic T1 Arabidopsis plants ectopically expressing sweet potato $S P C P 2$. A. Transition from vegetative growth to flowering was observed and compared 30 days after seed germination. B. The appearance and size of inflorescences and siliques were observed and compared 35 days after seed germination. C. RT-PCR analysis of SPCP2. C and AT denote control and transgenic T1 Arabidopsis plants, respectively. 


\subsection{Expression of sweet potato papain-like cysteine protease SPCP2 altered seed and silique development and enhanced stress tolerance in transgenic Arabidopsis}

Transgenic Arabidopsis plants were isolated and identified with floral dip transformation method (Clough \& Bent, 1998). Genomic PCR and RT-PCR analysis confirmed that the presence and expression of sweet potato papain-like cysteine protease SPCP2 in transgenic Arabidopsis plants (Chen et al., 2010). Transgenic Arabidopsis plants also exhibited slightly earlier transition from vegetative to reproductive growth (Fig. 5). The reasons and mechanisms are not clear. However, an imbalance in the relative availability of carbohydrate and nitrogen in the shoot apical meristem (Raper et al., 1988; Rideout et al., 1992), the change of endogenous ascorbic acid content (Barth et al., 2006), and possible nonspecific degradation and removal of flowering repressor(s) by ectopic SPCP2 expression are suggested.

Expression of sweet potato SPCP2 in transgenic Arabidopsis plants also caused elevated number of incompletely-developed silique (Fig. 6), and reduced average fresh weight per seed and lower germination percentage (Chen et al., 2010). The reasons for the altered phenotypic characteristics in transgenic Arabidopsis by ectopic SPCP2 gene expression are not clear. However, SPCP2 exhibited high amino acid sequence identities with plant papainlike cysteine proteases, such as Phaseolus vulgaris CP2, Vicia sativa CPR2, and Vigna mungo SH-EP. These papain-like cysteine proteases together with vacuolar processing enzymes have been implicated in association with the degradation and mobilization of globulin storage proteins during seed germination and seedling growth in Phaseolus vulgaris (Senyuk et al., 1998), Vigna mungo (Okamoto et al., 1999), and Vicia sativa (Schlereth et al., 2000; Schlereth et al., 2001). These reports provide a possible explanation for the altered phenotypic characteristics observed in transgenic Arabidopsis plants, and suggest that sweet potato SPCP2 may have an enzymatic function similar to papain-like cysteine proteases, including Vigna mungo SH-EP and Vicia sativa CPR2 for protein degradation and nutrient recycling during leaf senescence.

Expression of sweet potato SPCP2 in transgenic Arabidopsis plants exhibited higher salt and drought stress tolerance (Fig. 7), and contained higher relative water content than control (Fig. 8). The reasons for the altered stress responses in transgenic Arabidopsis by ectopic SPCP2 gene expression are not clear. However, SPCP2 exhibited high amino acid sequence identities with plant cysteine proteases, such as Arabidopsis RD19 and broccoli Bocp4. Arabidopsis RD19 was a drought-inducible cysteine protease (Koizumi et al., 1993), and belonged to osmotic stress-responsive genes (Xiong et al., 2002). Under osmotic stress such as drought, high salinity ( $\mathrm{NaCl}$ or $\mathrm{PEG}$ ) and ABA treatments, RD19 mRNA transcript was significantly enhanced compared to untreated control (Xiong et al., 2002). Broccoli Bocp4 exhibited high sequence identity to dehydration-responsive Arabidopsis RD19, and was also significantly induced in broccoli florets, which were kept in air (dry situation) but not in water or $2 \%$ sucrose solution $12 \mathrm{~h}$ post harvest (Coupe et al., 2003). Sweet potato cysteine protease SPCP2 was also inducible by salt and drought stresses in detached leaves (Fig. 9), and its ectopic expression in transgenic Arabidopsis caused higher salt and drought resistances (Figs. 7 and 8). Our results agree with these reports and suggest a possible role of sweet potato cysteine protease SPCP2 in osmotic stress regulation and salt/drought stress tolerance. 
A.

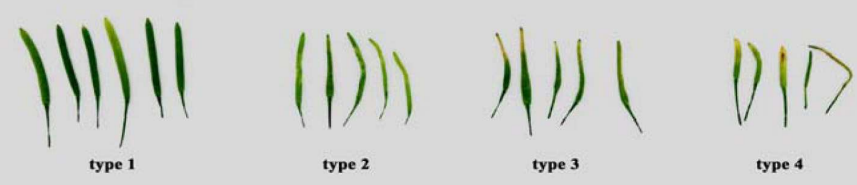

B.

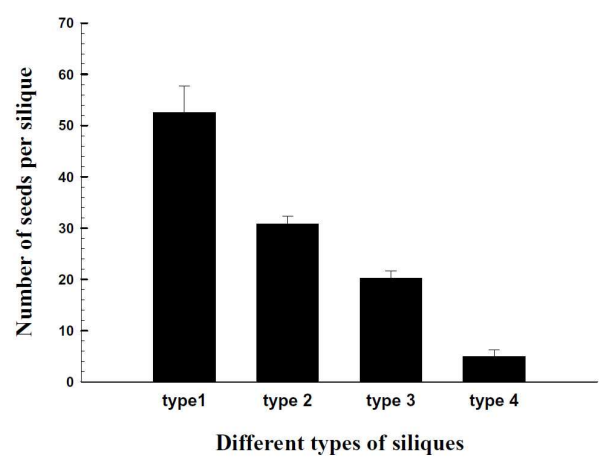

C.

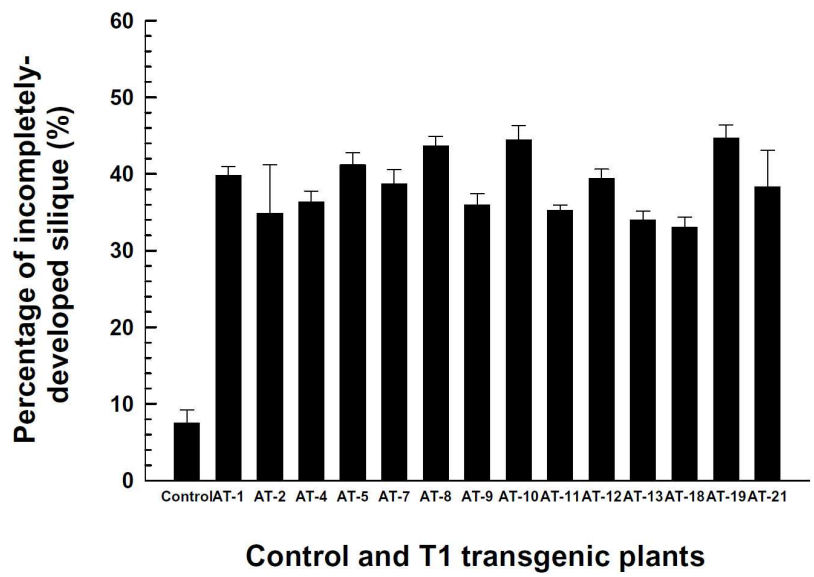

Fig. 6. Comparison of incompletely-developed silique percentages among control and transgenic T1 plants ectopically expressing sweet potato SPCP2. A. The appearance and size of different silique types (types 1, 2, 3 and 4) were observed and compared 35 days after seed germination. B. The average seed number of different silique type. C. Comparison of incompletely-developed silique percentage among control and transgenic T1 plants. $\mathrm{C}$ and AT denote control and transgenic T1 Arabidopsis plants,respectively (Adapted from Chen et al., 2010). 


\section{A. $\mathrm{NaCl}$}

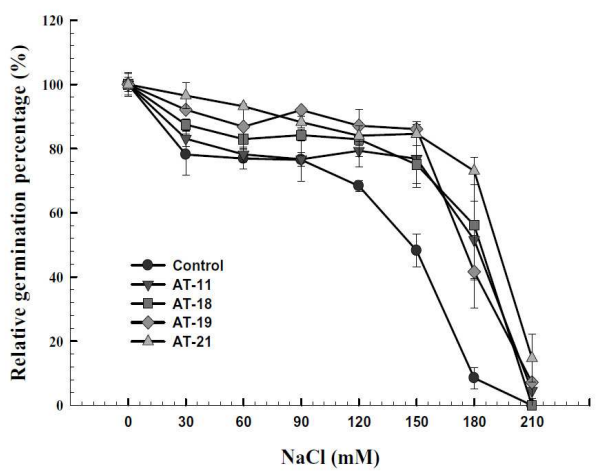

\section{B. Drought}
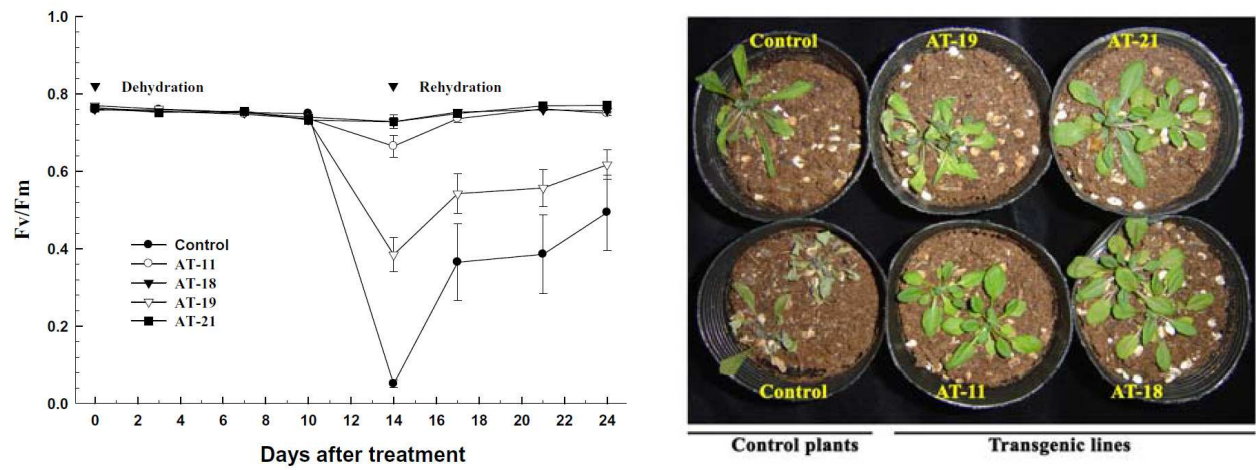

Drought stress at day 14

Fig. 7. Comparison of salt and drought stress tolerance among control and transgenic T1 Arabidopsis plants ectopically expressing sweet potato SPCP2. A. Salt. For salt treatment, seeds were germinated on half strength MS medium plus 3\% sucrose and different concentrations of $\mathrm{NaCl}$ for $\mathrm{ca} .2$ weeks, and the relative germination percentages were recorded and compared. B. Drought. For drought treatment, upper panel of B is the photochemical Fv/Fm comparison among control and transgenic T1 Arabidopsis plants during dehydration and rehydration treatment. Lower panel of $\mathrm{B}$ is the morphological comparison among control and transgenic T1 Arabidopsis plant at day 14 after drought treatment. The data were the average of total 5 petri dishes for A or 5 seedlings per transgenic line for B, and shown as mean \pm S.E. Control and AT-11/AT-18/AT-19/AT-21 denote wild type and transgenic T1 Arabidopsis plants, respectively. $\boldsymbol{\Delta}$ indicates the time points of dehydration and rehydration (Adapted from Chen et al., 2010). 


\section{Correlation of cysteine protease expression with storage protein degradation in sweet potato storage root during sprouting}

In sweet potato storage root, trypsin protease inhibitors (TIs) are the main storage proteins and composed of a multiple gene family. It has been implicated that cysteine proteases are likely associated with the degradation of storage root trypsin inhibitors during sprouting (Huang et al., 2005). Therefore, expression and correlation of sweet potato asparaginyl endopeptidase SPAE and papain-like cysteine protease SPCP2 with the degradation and mobilization of the two major storage root trypsin inhibitor bands during sprouting were studied. The sprouts appeared and were visible within the first week of incubation of storage root at room temperature, whereas, degradation of trypsin inhibitors became significant in the later incubation. RT-PCR analysis of SPAE and SPCP2 also demonstrated that their gene expression was significantly higher in the sprout and flesh of sprouting storage root than that of non-sprouting storage root (Fig. 10), and correlated well with the time course of degradation of the two major trypsin inhibitor bands (unpublished data). These data suggest that the asparaginyl endopeptidase SPAE and papain-like cysteine protease SPCP2 may play roles in association with storage root major trypsin inhibitor degradation during sprouting.

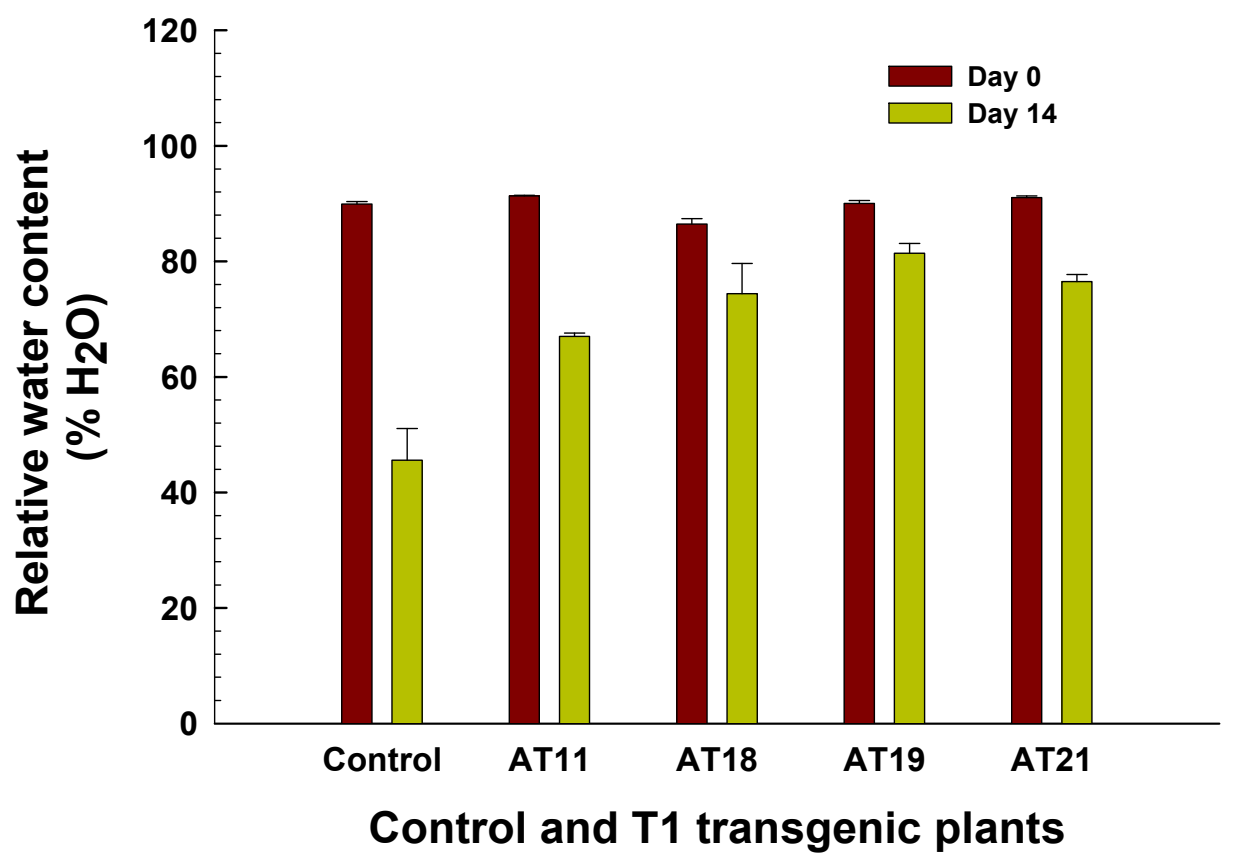

Fig. 8. Comparison of the relative water content $(\mathrm{H} 2 \mathrm{O} \%)$ between control and transgenic $\mathrm{T} 1$ Arabidopsis plants ectopically expressing sweet potato SPCP2 at day 14 after drought treatment. The data were the average of total 5 seedlings per transgenic plants, and shown as mean \pm S.E. Control and AT-11/AT-18/AT-19/AT-21 denote wild type and transgenic T1 Arabidopsis plants, respectively. 
A. $\mathrm{NaCl}$

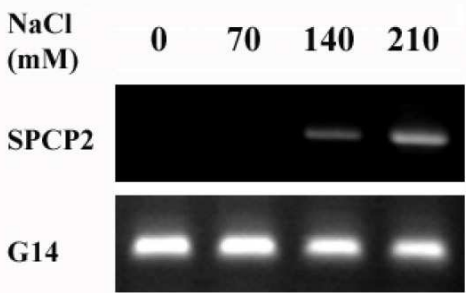

B. Drought

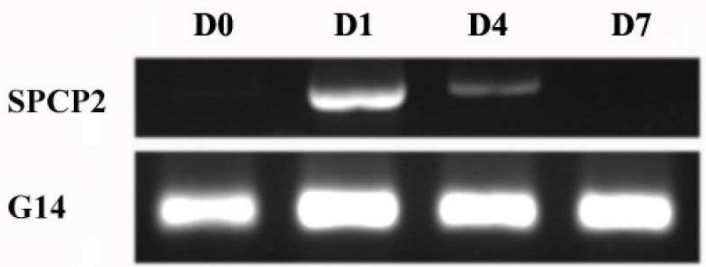

Fig. 9. Induction of sweet potato papain-like cysteine protease SPCP2 gene expression by salt and drought treatments. A. Salt treatment. Sweet potato detached leaves were treated with different salt concentrations $(0,70,140$ and $210 \mathrm{mM}$, respectively,) for 9 days and collected individually for RT-PCR analysis. B. Drought treatment. Detached sweet potato leaves were placed on dry paper tower in the dark for 0,1,4 and 7 days, and then collected individually for RT-PCR analysis. Sweet potato G14 encoded a constitutively expressed metallothionein-like protein and was used as a control.

A.

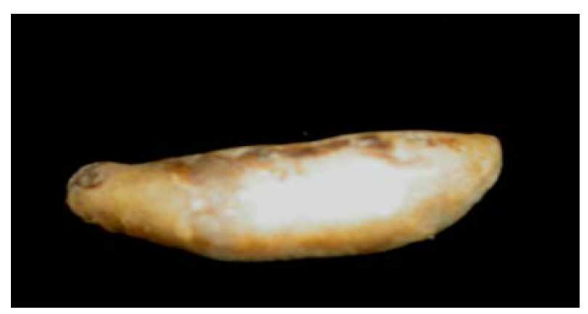

Non-sprouting storage root

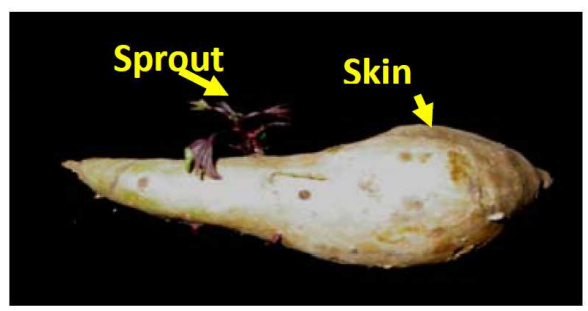

Sprouting storage root

B.

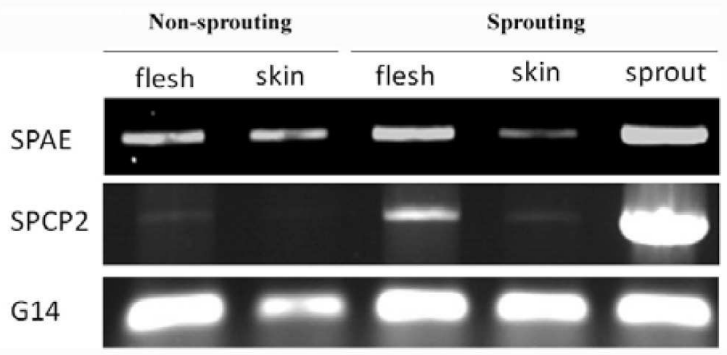

Fig. 10. Expression patterns of sweet potato asparaginyl endopeptidase SPAE and papainlike cysteine protease $S P C P 2$ in non-sprouting and sprouting st The orage roots. A. Storage root morphology. B. RT-PCR analysis of SPAE and SPCP2. Sweet potato G14 encoded a constitutively expressed metallothionein-like protein and used as a control. 
Sweet potato asparaginyl endopeptidase SPAE and papain-like cysteine protease SPCP2 cDNAs have been constructed and expressed in recombinant PET vector for fusion protein production and purification. Application of the purified fusion protein to sweet potato storage root or detached leaves will be performed in the future in order to study further whether they can promote (1) the degradation of storage root major trypsin inhibitors during sprouting, (2) protein degradation and recycling during leaf senescence, or (3) stress tolerance.

\section{Conclusion}

Sweet potato asparaginyl endopeptidase $S P A E$ and papain-like cysteine protease SPCP2 are senescence-associated genes and significantly enhanced their expression in senescent leaves. Phylogenetic tree analysis shows that SPAE and SPCP2 exhibit close association with vacuolar processing enzyme and papain-like cysteine protease, respectively, which are involved in seed globulin storage protein degradation. Ectopic expression of sweet potato SPAE and SPCP2 in transgenic Arabidopsis plants also caused altered phenotypic characteristics, including abnormal seed and silique development, elevated incompletelydeveloped silique percentage, fewer silique numbers per plant, reduced seed germination percentage, and enhanced tolerance to drought and salt stresses. Based on these data, it can be concluded that sweet potato asparaginyl endopeptidase $S P A E$ and papain-like cysteine protease SPCP2 may play physiological roles in association with protein degradation and nutrient recycling during leaf senescence with enzymatic functions similar to seed globulin storage protein degradation and re-mobilization during germination and seedling growth.

\section{References}

Abe, Y., Shirane, K., Yokosawa, H., Matsushita, H., Mitta, M., Kato, I. \& Ishii, S. (1993). Asparaginyl endopeptidases of jack bean seeds. J. Biol. Chem., 268, 3523-3529

Barth, C., De Tullio, M. \& Conklin, P.L. (2006). The role of ascorbic acid in the control of flowering time and the onset of senescence. J. Exp. Bot., 57, 1657-1665

Bernoux, M., Timmers, T., Jauneau, A., Brie, C., de Wit, P.J.G.M., Marco, Y. \& Deslandesa, L. (2008). RD19, an Arabidopsis cysteine protease required for RRS1-R-mediated resistance, is relocalized to the nucleus by the Ralstonia solanacearum PopP2 effector. Plant Cell, 20, 2252-2264

Buchanan-Wollaston, V. (1997). The molecular biology of leaf senescence. J. Exp. Bot., 48, 181-199

Buchanan-Wollaston, V., Page, T., Harrison, E., Breeze, E. \& Lim, P.O. (2005). Comparative transcriptome analysis reveals significant differences in gene expression and signaling pathways between developmental and dark/starvationinduced senescence in Arabidopsis. Plant J. 42, 567-585

Chen, H.J., Hou, W.C., Jane, W.N. \& Lin, Y.H. (2000). Isolation and characterization of an isocitrate lyase gene from senescent leaves of sweet potato (Ipomoea batatas cv. Tainong 57). J. Plant Physiol., 157, 669-676 
Chen, H.J., Hou, W.C., Yang, C.Y., Huang, D.J., Liu, J.S. \& Lin, Y.H. (2003). Molecular cloning of two metallothionein-like protein genes with differential expression patterns from sweet potato (Ipomoea batatas (L.) Lam.) leaves. J. Plant Physiol., 160, 547-555

Chen, H.J., Hou, W.C., Liu, J.S., Yang, C.Y., Huang, D.J. \& Lin, Y.H. (2004). Molecular cloning and characterization of a cDNA encoding asparaginyl endopeptidase from sweet potato (Ipomoea batatas (L.) Lam) senescent leaves. J. Exp. Bot., 55, 825835

Chen, H.J., Huang, G.J., Hou, W.C., Liu, J.S. \& Lin, Y.H. (2006). Molecular cloning and characterization of a granulin-containing cysteine protease SPCP3 from sweet potato (Ipomoea batatas) senescent leaves. J. Plant Physiol., 163, 863-876

Chen, H.J., Wen, I.C., Huang, G.J., Hou, W.C. \& Lin, Y.H. (2008). Expression of sweet potato asparaginyl endopeptidase caused altered phenotypic characteristics in transgenic Arabidopsis. Bot. Stud., 49, 109-117

Chen, H.J., Huang, G.J., Chen, W.S., Su, C.T., Hou W.C. \& Lin, Y.H. (2009). Molecular cloning and expression of a sweet potato cysteine protease SPCP1 from senescent leaves. Bot. Stud., 50, 159-170.

Chen, H.J., Tsai, Y.J., Chen, W.S., Huang, G.J., Huang, S.S. \& Lin, Y.H. (2010a). Ethephonmediated effects on leaf senescence are affected by reduced glutathione and EGTA in sweet potato detached leaves. Bot. Stud., 51, 171-181

Chen, H.J., Su, C.T., Lin, C.H., Huang, G.J. \& Lin, Y.H. (2010b). Expression of sweet potato cysteine protease SPCP2 altered developmental characteristics and stress responses in transgenic Arabidopsis plants. J. Plant Physiol., 167, 838-847

Chen, J.M., Rawlings, N.D., Stevens, R.A.E. \& Barrett, A.J. (1998). Identification of the active site of legumain links it to caspases, clostripain and gingipains in a new clad of cysteine endopeptidases. FEBS Lett., 441, 361-365

Chrispeels, M.J. \& Herman, E.M. (2000). Endoplasmic reticulum-derived compartments function in storage and as mediators of vacuolar remodeling via a new type of organelle, precursor protease vesicles. Plant Physiol., 123, 1227-1233.

Clough, S.J. \& Bent, A.F. (1998). Floral dip: a simplified method for Agrobacteriummediated transformation of Arabidopsis thaliana. Plant J., 16, 735-743

Coupe, S.A., Sinclair, B.K., Watson, L.M., Heyes, J.A. \& Eason. J.R. (2003). Identification of dehydration-responsive cysteine proteases during post-harvest senescence of broccoli florets. J. Exp. Bot., 54, 1045 - 1056

Gruis, D.F., Selinger, D.A., Curran, J.M. \& Jung, R. (2002). Redundant proteolytic mechanisms process seed storage proteins in the absence of seed-type members of the vacuolar processing enzyme family of cysteine proteases. Plant Cell, 14, 2863-2882

Hara-Nishimura, I., Inoue, K. \& Nishimura, M. (1991). A unique vacuolar processing enzyme responsible for conversion of several proprotein precursors into the mature forms. FEBS Lett., 294, 89-93

Hara-Nishimura, I., Shimada, T., Hiraiwa, N. \& Nishimura, M. (1995). Vacuolar processing enzyme responsible for maturation of seed proteins. J. Plant Physiol., $145,632-640$ 
Huang, D.J., Chen, H.J., Hou, W.C., Chen, T.E., Hsu, W.Y. \& Lin, Y.H. (2005). Expression and function of a cysteine proteinase cDNA from sweet potato (Ipomoea batatas [L.] Lam 'Tainong 57') storage roots. Plant Sci., 169, 423-431

Koizumi, M., Yamaguchi-Shinozaki, K. \& Shinozaki, K. (1993). Structure and expression of two genes that encode distinct drought-inducible cysteine proteinases in Arabidopsis thaliana. Gene, 129, 175-182

Kuroyanagi, M., Nishimura, M. \& Hara-Nishimura, I. (2002). Activation of Arabidopsis vacuolar processing enzyme by self-catalytic removal of an auto-inhibitory domain of the C-terminal propeptide. Plant Cell Physiol., 43, 143-151

Lim, P.O., Kim, H.J. \& Nam, H.G. (2007). Leaf senescence. Annu. Rev. Plant Biol., 58, 115136

Lin, C.H. (2010). Ectopic expression of sweet potato cysteine protease SPCP2 promotes earlier flowering and enhances drought stress tolerance. Master thesis, Department of Biological Sciences, National Sun Yat-sen University, Kaohsiung, Taiwan

Makino, A. \& Osmond, B. (1991). Effects of nitrogen nutrition on nitrogen partitioning between chloroplasts and mitochondria in pea and wheat. Plant Physiol., 96, 355362

Martı́nez, D.E., Costa, M.L., Gomez, F.M., Otegui, M.S. \& Guiamet, J.J. (2008). 'Senescence-associated vacuoles' are involved in the degradation of chloroplast proteins in tobacco leaves. Plant J., 56, 196-206

Marty, F. (1999). Plant vacuoles. Plant Cell, 11, 587-599

Mitsuhashi, W., Koshiba, T. \& Minamikawa, T. (1986). Separation and characterization of two endopeptidases from cotyledons of germinating Vigna mungo seeds. Plant Physiol., 80, 628-634

Müntz, K. \& Shutov, A.D. (2002). Legumains and their functions in plants. Trends in Plant Sci., 7, 340-344

Okamoto, T. \& Minamikawa, T. (1995). Purification of a processing enzyme (VmPE-1) that is involved in post-translational processing of a plant cysteine endopeptidase (SH-EP). Eur. J. Biochem., 231, 300-305

Okamoto, T. \& Minamikawa, T. (1999). Molecular cloning and characterization of Vigna mungo processing enzyme 1 (VmPE-1), an asparaginyl endopeptidase possibly involved in post-translational processing of a vacuolar cysteine endopeptidase (SH-EP). Plant Mol. Biol., 39, 63-73

Okamoto, T., Yuki, A., Mitsuhashi, N. \& Mimamikawa, T. (1999). Asparaginyl endopeptidase (VmPE-1) and autocatalytic processing synergistically activate the vacuolar cysteine proteinase (SH-EP). Eur. J. Biochem., 264, 223-232

Okamoto, T., Shimada, T., Hara-Nishimura, I., Nishimura, M. \& Minamikawa, T. (2003). C-terminal KDEL sequence of A KDEL-tailed cysteine proteinase (sulfhydrylendopeptidase) is involved in formation of KDEL vesicle and in efficient vacuolar transport of sulfhydryl-endopeptidase1. Plant Physiol., 132, 1892-1900

Poueymiro, M. \& Genin, S.P. (2009). Secreted proteins from Ralstonia solanacearum: a hundred tricks to kill a plant. Curr. Opin. Microbiol., 12, 44-52 
Quirino, B.F., Noh, Y.S., Himelblau, E. \& Amasino, R.M. (2000). Molecular aspects of leaf senescence. Trends Plant Sci., 5, 278-282

Raper, C.D.J., Thomas, J.F., Tolley-Henry, L. \& Rideout, J.W. (1988). Assessment of an apparent relationship between availability of soluble carbohydrates and reduced nitrogen during floral initiation in tobacco. Bot. Gaz., 149, 289-294

Rideout, J.W., Raper, C.D. \& Miner, G.S. (1992). Changes in ratio of soluble sugars and free amino nitrogen in the apical meristem during floral transition of tobacco. Int. J. Plant Sci., 153, 78-88

Rojo, E., Zouhar, J., Carter, C., Kovaleva, V. \& Raikhel, N.V. (2003). A unique mechanism for protein processing and degradation in Arabidopsis thaliana. Proc. Natl. Acad. Sci. USA, 100, 7389-7394

Schlereth, A., Becker, C., Horstmann, C., Tiedemann, J. \& Muntz, K. (2000). Comparison of globulin mobilization and cysteine proteinases in embryonic axes and cotyledons during germination and seedling growth of vetch (Vicia sativa L.). J. Exp. Bot., 51, 1423-1433

Schlereth, A., Standhardt, D., Mock, H.P. \& Muntz, K. (2001). Stored cysteine proteinases start globulin mobilization in protein bodies of embryonic axes and cotyledons during vetch (Vicia sativa L.) seed germination. Planta, 212, 718-727

Scott, M.P., Jung, R., Muntz, K. \& Nielsen, N.C. (1992). A protease responsible for posttranslational cleavage of a conserved Asn-Gly linkage in glycinin, the major seed storage protein of soybean. Proc. Natl. Acad. Sci. USA, 89, 658-662

Senyuk, V., Rotari, V., Becker, C., Zakharov, A., Horstmann, C., Muntz, K. \& Vaintraub, L. (1998). Does an asparaginyl-specific cysteine endopeptidase trigger phaseolin degradation in cotyledons of kidney bean seedlings? Eur. J. Biochem., 258, 546558

Shimada, T., Hiraiwa, N., Nishimura, M. \& Hara-Nishimura, I. (1994). Vacuolar processing enzyme of soybean that converts proproteins to the corresponding mature forms. Plant Cell Physiol., 35, 713-718

Shimada, T., Yamada, K. \& Kataoka, M. (2003). Vacuolar processing enzymes are essential for proper processing of seed storage proteins in Arabidopsis thaliana. J. Biol. Chem., 278, 32292-32299

Tiedemann, J., Schlereth, A. \& Müntz, K. (2001). Differential tissue-specific expression of cysteine proteinases forms the basis for the fine-tuned mobilization of storage globulin during and after germination in legume seeds. Planta, 212, 728738

Toyooka, K., Okamoto, T. \& Minamikawa, T. (2000). Mass transport of proform of a FDEL-tailed cysteine proteinase (SH-EP) to protein storage vacuoles by endoplasmic reticulum-derived vesicle is involved in protein mobilization in germinating seeds. J. Cell Biol., 148, 453-463

Vierstra, R.D. (1996). Proteolysis in plants: mechanisms and functions. Plant Mol. Biol., 32, 275-302

Xiong, L., Lee, H., Ishitani, M. \& Zhu, J.K. (2002). Regulation of osmotic stress-responsive gene expression by the LOS6/ABA1 locus in Arabidopsis. J. Biol. Chem., 277, 85888596 
Yoshida, S. (2003). Molecular regulation of leaf senescence. Curr. Opin. Plant Biol., 6, 79-84 


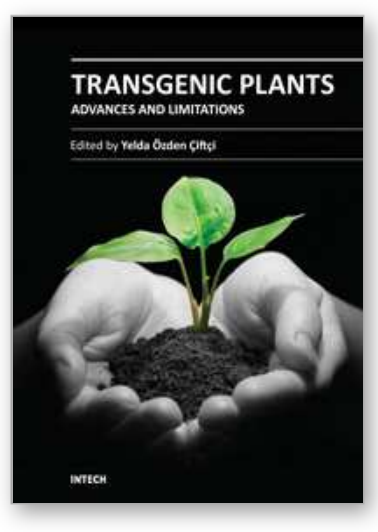

\author{
Transgenic Plants - Advances and Limitations \\ Edited by PhD. Yelda Ozden Çiftçi
}

ISBN 978-953-51-0181-9

Hard cover, 478 pages

Publisher InTech

Published online 07, March, 2012

Published in print edition March, 2012

Development of efficient transformation protocols is becoming a complementary strategy to conventional breeding techniques for the improvement of crops. Thus, Transgenic Plants - Advances and Limitations covers the recent advances carried on improvement of transformation methods together with assessment of the impact of genetically transformed crops on biosafety. Each chapter has been written by one or more experienced researchers in the field and then carefully edited to ensure throughness and consistency.

\title{
How to reference
}

In order to correctly reference this scholarly work, feel free to copy and paste the following:

Hsien-Jung Chen, Guan-Jhong Huang, Chia-Hung Lin, Yi-Jing Tsai, Zhe-Wei Lin, Shu-Hao Liang and YawHuei Lin (2012). Expression of Sweet Potato Senescence-Associated Cysteine Proteases Affect Seed and Silique Development and Stress Tolerance in Transgenic Arabidopsis, Transgenic Plants - Advances and Limitations, PhD. Yelda Ozden Çiftçi (Ed.), ISBN: 978-953-51-0181-9, InTech, Available from:

http://www.intechopen.com/books/transgenic-plants-advances-and-limitations/expression-of-sweet-potatosenescence-associated-cysteine-proteases-affect-seed-and-silique-developm

\section{INTECH}

open science | open minds

\section{InTech Europe}

University Campus STeP Ri

Slavka Krautzeka 83/A

51000 Rijeka, Croatia

Phone: +385 (51) 770447

Fax: +385 (51) 686166

www.intechopen.com

\section{InTech China}

Unit 405, Office Block, Hotel Equatorial Shanghai

No.65, Yan An Road (West), Shanghai, 200040, China

中国上海市延安西路65号上海国际贵都大饭店办公楼405单元

Phone: +86-21-62489820

Fax: +86-21-62489821 
(C) 2012 The Author(s). Licensee IntechOpen. This is an open access article distributed under the terms of the Creative Commons Attribution 3.0 License, which permits unrestricted use, distribution, and reproduction in any medium, provided the original work is properly cited. 